\title{
NIOBIUM CAVITY DEVELOPMENT FOR THE HIGH-ENERGY LINAC OF THE RARE ISOTOPE ACCELERATOR
}

\author{
C. C. Compton, T. L. Grimm, W. Hartung, H. Podlech, R. C. York \\ National Superconducting Cyclotron Lab, ${ }^{*}$ Michigan State University, East Lansing, Michigan \\ G. Ciovati, P. Kneisel \\ Thomas Jefferson National Accelerator Facility, Newport News, Virginia \\ D. Barni, C. Pagani, P. Pierini \\ INFN Milano-Laboratorio LASA, 20090 Segrate (Milano), Italy
}

\section{INTRODUCTION}

The Rare Isotope Accelerator (RIA) is being designed to supply an intense beam of exotic isotopes for nuclear physics research [1]. Superconducting cavities are to be used to accelerate the $\mathrm{CW}$ beam of heavy ions to $400 \mathrm{MeV}$ per nucleon, with a beam power of up to $400 \mathrm{~kW}$. Because of the varying velocity of the ion beam along the linac, a number of different types of superconducting structures are needed.

The RIA linac will accelerate heavy ions over the same velocity range as the proton linac for the Spallation Neutron Source (SNS). It was decided to use the 6-cell axisymmetric $805 \mathrm{MHz}$ cavities and cryostats of SNS for the downstream portion of the RIA linac, thereby saving the non-recurring development and engineering costs. For additional cost saving, it was decided to extend the SNS multi-cell axisymmetric cavity design to lower velocity, $\beta=v / c=0.4$, using the same cryostats and RF systems. Axisymmetric cavities will thus constitute about three-quarters of RIA's total accelerating voltage, and most of that voltage will be provided by cavities already developed for SNS.

The axisymmetric cavities will accelerate the RIA beam from $\beta=0.4$ to $\beta=0.72$. This velocity range can be efficiently covered with two different types of 6-cell cavities, one with a geometric $\beta, \beta_{g}$, of 0.47 , and the other with a $\beta_{g}$ of 0.61 . The $\beta_{g}=0.61$ cavity will be of the existing SNS design; some $\beta_{g}=0.81$ SNS cavities may also be desired at the end of the RIA linac for acceleration of light ions above $400 \mathrm{MeV}$ per nucleon. Prototypes for both $\beta_{g}=0.61$ and $\beta_{g}=0.81$ have been fabricated and tested [2]. The $\beta_{g}=0.47$ cavity is the focus of the present work.

The reduction in $\beta_{g}$ to 0.47 results in less favourable electromagnetic and mechanical properties, and opens up the possibility of multipacting, but several groups have already designed and prototyped cavities in this range. These efforts have been motivated mostly by the interest in highintensity proton linacs that has arisen in the past 8 years. Single-cell cavities for $\beta=0.47$ to 0.5 have been tested at various laboratories $[3,4,5]$. The gradients and $Q$ 's reached in the single-cell tests have exceeded the design goals, and multipacting has not limited the performance.

This paper will cover the design of a 6 -cell $\beta_{g}=0.47$

\footnotetext{
* Supported by the National Science Foundation and Michigan State University.
}

cavity for RIA, as well as the fabrication and RF testing of single-cell prototypes. Single-cell prototypes were chosen as a first step, as they provide a quick and inexpensive way to find out whether the desired field level and $Q$ can be reached, and to check for problems with multipacting. An accelerating gradient of $8 \mathrm{MV} / \mathrm{m}$ was chosen as a goal for the $\beta_{g}=0.47$ cavity.

\section{SIX-CELL CAVITY DESIGN}

The optimised design for the 6-cell $\beta_{g}=0.47$ cavity for RIA is presented in [6]. Table 1 gives the primary parameters of the structure, and Figure 1 shows the cross section of the cavity with the electric field lines for the accelerating mode. With a gradient $\left(E_{a}\right)$ of $8 \mathrm{MV} / \mathrm{m}$, the peak surface electric field $\left(E_{p}\right)$ is below $27 \mathrm{MV} / \mathrm{m}$, the value chosen for the design of the SNS cavities, and the peak surface magnetic field $\left(B_{p}\right)$ is below $60 \mathrm{mT}$. Note that $E_{a}$ includes the transit time of a particle with the optimum $\beta$, so that $E_{a}=8 \mathrm{MV} / \mathrm{m}$ corresponds to a voltage gain of $4.2 \mathrm{MV}$ per cavity for a particle with the optimum $\beta$ accelerated on crest. The cell-to-cell coupling factor is $1.5 \%$, which is the same as for the SNS cavities. For mechanical stability (and practical etching and rinsing), the inclination of the cell wall is $\geq 6.5^{\circ}$. The Lorentz detuning coefficient, $K_{L}=d f / d E_{a}^{2}$, is nevertheless quite high, even with stiffening $\left(-13.7 \mathrm{~Hz} /(\mathrm{MV} / \mathrm{m})^{2}\right.$ for a wall thickness of $\left.3.8 \mathrm{~mm}\right)$. Lorentz detuning should not be a problem in itself, since RIA is a CW machine, but microphonics may be an issue due to the relatively low beam loading. Calculations indicate that multipacting problems are unlikely for the chosen

Table 1. Parameters for 6-cell cavity; $R_{S}$ is the shunt impedance (linac definition). RF quantities were calculated with SUPERFISH [7].

\begin{tabular}{|l|c|}
\hline Mode & $T M_{010} \pi$ \\
\hline Resonant frequency $f$ & $805 \mathrm{MHz}$ \\
\hline Cell-to-cell coupling & $1.5 \%$ \\
\hline$E_{p} / E_{a}$ & 3.41 \\
\hline$c B_{p} / E_{a}$ & 2.07 \\
\hline$R_{s} / Q$ & $160 \Omega$ \\
\hline Geometry factor & $136.7 \Omega$ \\
\hline \hline Active length & $527 \mathrm{~mm}$ \\
\hline Inner diameter at iris (aperture) & $77.2 \mathrm{~mm}$ \\
\hline Inner diameter at equator & $329 \mathrm{~mm}$ \\
\hline
\end{tabular}




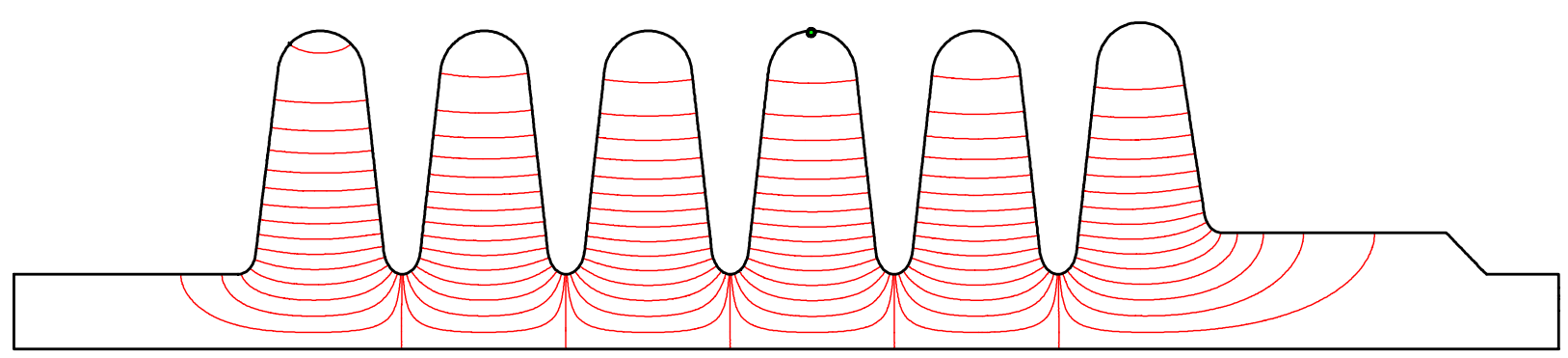

Figure 1. Electric field lines from SUPERFISH [7] for the $T M_{010} \pi$ mode in the 6-cell $\beta_{g}=0.47$ cavity.

geometry, because the electrons do not acquire enough energy to generate secondary electrons when impacting on the surface.

The $\beta_{g}=0.47$ cell shape is compared with those of the SNS cavities and the TeSLA Test Facility (TTF) cavity [8] in Figure 2. As can be seen, the $\beta_{g}=0.47$ cell shape is relatively close to the $\beta_{g}=0.61$ cell shape. Thus, stiffening and bracing techniques being developed for the SNS cavities should be applicable to the $\beta_{g}=0.47$ cavity.

As shown in Figure 1, the present $\beta_{g}=0.47$ design incorporates an enlarged beam tube at one end to accommodate the input coupler, as for the SNS cavities. This enlarged beam pipe may not be necessary for RIA, due to the comparatively low beam power. This could lead to additional savings in the fabrication cost.

As discussed above, the RIA cavities must accelerate a beam whose velocity is varying with position. The normalised transit time factor of the 6-cell $\beta_{g}=0.47$ cavity is shown in Figure 3 . The cavity will be used from $\beta=0.4$ to $\beta=0.53$ (indicated by dotted lines in Figure 3 ). The choice of 6 cells is a reasonable compromise between a long structure to provide higher voltage and a small number of cells for higher velocity acceptance. Note that the maximum energy is imparted to particles with $\beta$ slightly larger than $\beta_{g}$, as they acquire a little more energy travelling through the cavity and suffer less deceleration from the evanescent field

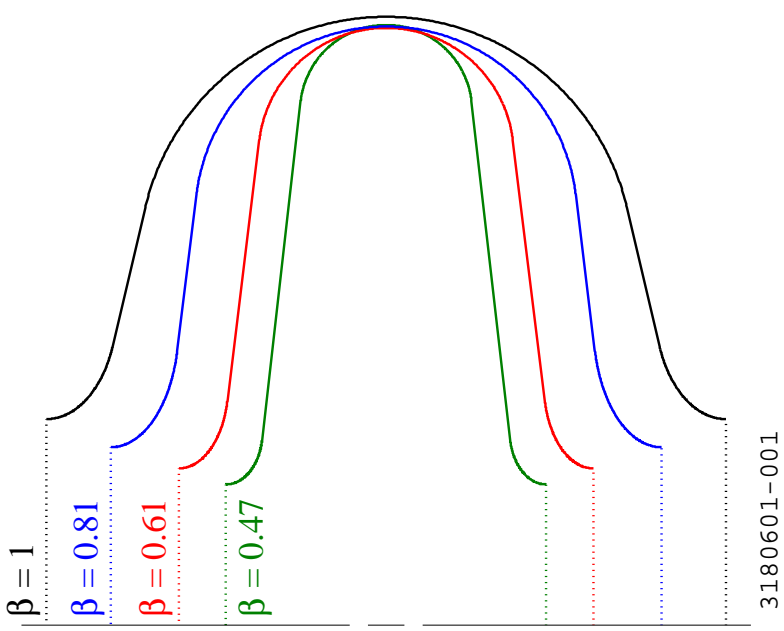

Figure 2. Comparison of the $\beta_{g}=0.47$ cell with a $\beta_{g}=$ 0.61 cell (SNS), a $\beta_{g}=0.81$ cell (SNS), and a $\beta_{g}=1$ cell (TTF, scaled to $805 \mathrm{MHz}$ ).

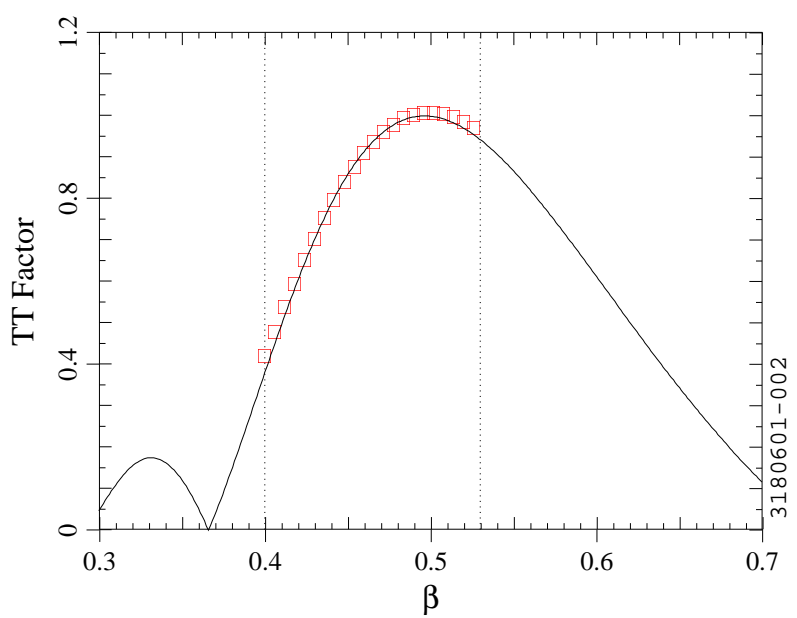

Figure 3. Dependence of the normalised transit time factor on $\beta$ for the $\beta_{g}=0.47$ cavity. The squares are calculated via integration of the SUPERFISH electric field; the solid line is based on an analytic approximation (adjusted to better match the SUPERFISH curve).

in the beam tube. This effect becomes more pronounced as the number of cells is reduced.

\section{SINGLE-CELL CAVITY FABRICATION}

Two single-cell prototypes of the $\beta_{g}=0.47$ cavity have been fabricated. Sheet $\mathrm{Nb}$ of thickness $4 \mathrm{~mm}$ and of nominal Residual Resistivity Ratio (RRR) of 250 was used. The half-cells were deep drawn using $\mathrm{Al}$ alloy dies, following the same procedures as used for SNS cavities. The beam tubes were stamped from $3.2 \mathrm{~mm}$ thick reactor grade $\mathrm{Nb}$ sheet. The half-cells, beam tubes, and flanges were joined by electron beam welding. $\mathrm{Nb}$-Ti flanges were used for better surface hardness, so that an $\mathrm{Al}-\mathrm{Mg}$ gasket can be used for the vacuum seal.

The completed single-cell cavities were etched with the traditional Buffered Chemical Polishing solution to remove a layer of material from the inner surface. About $60 \mu \mathrm{m}$ was removed from the surface of the first cavity (additional chemistry was done after the first RF test, see below), and about twice that amount was removed from the second cavity. Following the etch, the cavity was brought into a Class 100 clean room, a high pressure water rinse was done with ultra-pure water, and the cavity was sealed and pumped out. It was necessary to attach the cavity to a rigid frame prior 


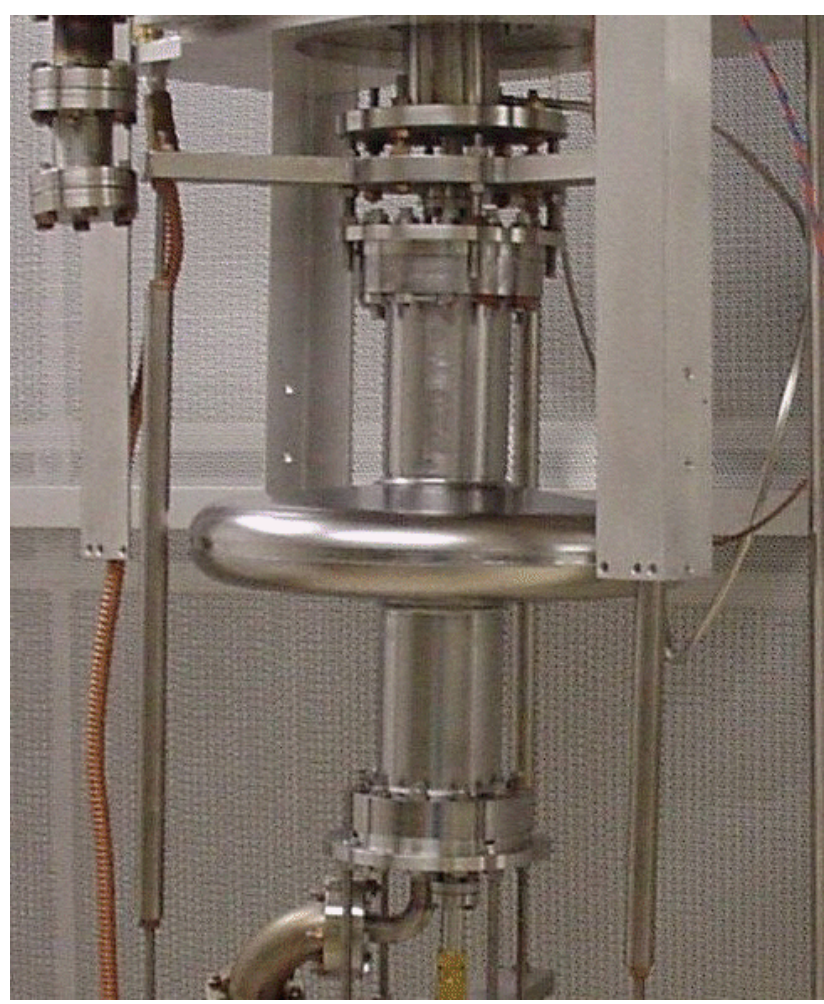

Figure 4. Photograph of a single-cell $\beta_{g}=0.47$ cavity on the RF test stand.

to the pump-out in order to prevent collapse under the pressure differential due to the vacuum inside. Figure 4 shows the first cavity after attachment to the insert for the RF test.

\section{RF TESTS}

The first single-cell $\beta_{g}=0.47$ cavity was tested twice in a vertical dewar configuration; the second cavity was tested once. In the first test of the first cavity, the $Q$ at $2 \mathrm{~K}$ and at low field was about $10^{10}$; a gradient of $E_{a}=4 \mathrm{MV} / \mathrm{m}$ was reached, with the $Q$ dropping significantly (to about $10^{9}$ ) at this field level. It appeared that there was some sort of barrier at $E_{a}=4 \mathrm{MV} / \mathrm{m}$, although the radiation was not very intense.

After the first RF test, it was suspected that the etch had not removed enough material, so another etch was done on the first cavity to remove an additional $\sim 40 \mu \mathrm{m}$ of material, and the high-pressure rinse and RF test were repeated. The measurement of $Q$ as a function of field level is shown in Figure 5. The results of the test on the second cavity are also shown. As can be seen, the low-field $Q$ was between $3 \cdot 10^{10}$ and $4 \cdot 10^{10}$ and the $Q$ remained above $10^{10}$ up to $E_{a}=15 \mathrm{MV} / \mathrm{m}$. The power dissipation in the cavity was about $0.6 \mathrm{~W}$ at $E_{a}=8 \mathrm{MV} / \mathrm{m}$. Gradients of 15.5 and $15 \mathrm{MV} / \mathrm{m}$ were reached with the first and second cavities, respectively. No high-field quench was observed, so both cavities could presumably have reached higher gradients. The low-field $Q$ at $2 \mathrm{~K}$ corresponds to a surface resistance of about $4 \mathrm{n} \Omega$ (note that, for this frequency, the BCS contribution is still significant at $2 \mathrm{~K}$ ).

The rate of change in resonant frequency $f$ as a function of pressure $P$ was measured during the cool-down from 4.2

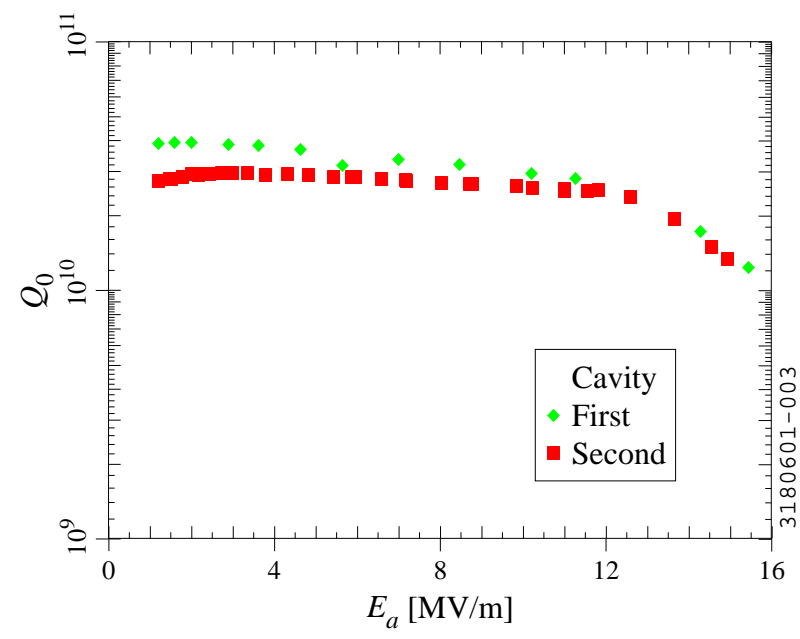

Figure 5. Measured dependence of $Q$ on accelerating gradient at $2 \mathrm{~K}$ for $\beta_{g}=0.47$ single-cell cavities.

$\mathrm{K}$ to $2 \mathrm{~K}$, with the result $d f / d P=-1.0 \mathrm{kHz} /$ torr. The shift in frequency as a function of field level was measured at 2 $\mathrm{K}$, with the result $K_{L}=-22 \mathrm{~Hz} /(\mathrm{MV} / \mathrm{m})^{2}$. The measured surface resistance at $4.2 \mathrm{~K}$ was $238 \mathrm{n} \Omega$ and $233 \mathrm{n} \Omega$ for the first and second cavities, respectively.

\section{CONCLUSION}

The RF tests on the single-cell prototypes have exceeded the desired accelerating gradient by almost a factor of 2 , with a $Q>10^{10}$ measured at the design gradient. This provides a good proof of principle for the RF performance of the $\beta_{g}=0.47$ cavity and demonstrates that multipacting is not a problem for this cell shape. The single-cell results are promising enough for the fabrication of a multi-cell prototype as the next step. Microphonics measurements on the single-cell and multi-cell cavities are also of interest.

\section{REFERENCES}

[1] K. W. Shepard et al., in 9th Workshop on RF Superconductivity: Proceedings, Report LA-13782-C, LANL, Los Alamos, New Mexico, 2000, p. 345-351.

[2] P. Kneisel et al., "Superconducting Prototype Cavities for the Spallation Neutron Source (SNS) Project," these proceedings.

[3] W. B. Haynes et al., in Proceedings of the Eighth Workshop on RF Superconductivity Report LNL-INFN (Rep) 133/98, Laboratori Nazionali di Legnaro, Legnaro (Padova), Italy, 1998, p. 523-533.

[4] Kenji Saito et al., Ibid., p. 534-539.

[5] Carlo Pagani et al., "Status of the High Energy SC Linac for the TRASCO Program," these proceedings.

[6] D. Barni et al., Tech Note JLab-TN-01-014, Jefferson Lab, Newport News, Virginia (2001).

[7] K. Halbach \& R. F. Holsinger, Particle Accelerators 7, p. 213-222 (1976).

[8] B. Aune et al., Physical Review Special Topics-Accelerators and Beams 3, 092001 (2000). 